\title{
Finite-Time Passivity of Stochastic Coupled Complex Networks
}

\author{
Xunwu Yin $\mathbb{D D}^{1}$ and Min $\mathrm{Cao}^{2}$ \\ ${ }^{1}$ School of Mathematical Sciences, Tiangong University, Tianjin 300387, China \\ ${ }^{2}$ School of Computer Science and Technology, Tiangong University, Tianjin 300387, China \\ Correspondence should be addressed to Xunwu Yin; yinxunwu@163.com
}

Received 30 April 2021; Accepted 13 June 2021; Published 9 July 2021

Academic Editor: A. E. Matouk

Copyright (C) 2021 Xunwu Yin and Min Cao. This is an open access article distributed under the Creative Commons Attribution License, which permits unrestricted use, distribution, and reproduction in any medium, provided the original work is properly cited.

\begin{abstract}
The finite-time passivity problem is, respectively, investigated for stochastic coupled complex networks (SCCNs) with and without time-varying delay. Firstly, we present several new concepts about finite-time passivity in the sense of expectation on the basis of existing passivity definition. By designing appropriate controllers, the finite-time passivity of SCCNs with and without timevarying delay is obtained. In addition, the definition of finite-time synchronization in the sense of expectation is proposed. Under some sufficient conditions and designed controllers, finite-time passivity derives finite-time synchronization. Finally, two examples are given to demonstrate the effectiveness of finite-time passive and synchronization criteria.
\end{abstract}

\section{Introduction}

In the real world, complex networks can be seen everywhere such as food webs, communication networks, World Wide Web, and many others [1-3]. Due to various uncertainties in the actual system, complex network systems may be affected by noise. In recent years, the stability of stochastic systems has been extensively studied. At the same time, the synchronization and stability of stochastic complex networks have gradually become a topic of widespread concern for scholars in various fields [4-8].

Passivity is one part of dissipativeness. The main property of passivity is keeping the systems internally stable. The passivity theory has been extensively applied in many fields such as stability, complexity, signal processing, chaos control, synchronization fuzzy control, and so on [9-12]. These are the main reasons why the passivity theory has been one of the most active research areas. In [13], the problem of passivity analysis was studied for discrete-time stochastic Markovian jump neural networks with both discrete and distributed delays. In [14], the problem of passivity analysis is investigated for a class of discrete-time stochastic neural networks with time-varying delays.
It is well known that passivity theory can provide a powerful tool to analyze synchronization of complex networks. However, in many existing works, synchronization is defined over the infinite time interval. Most of the theoretical methods on the synchronization of complex networks can only realize the network [15] or exponential asymptotical synchronization [16] which guarantees that error tends to 0 when $t$ tends to infinity. That is to say, achieving asymptotically stable convergence will be in infinite time. No further consideration has been given to the time and speed of synchronization. However, in practical engineering, people usually expect faster convergence rate and predict the required convergence time. Consequently, in order to achieve better control, the idea of finite-time synchronization has been proposed, and more and more attention has been paid by researchers. This kind of method can predict the synchronization time in advance and has better robustness, anti-interference, and better control effect. It has important research significance in theory and practice. Therefore, it is more meaningful to study finite-time synchronization [17-21]. In [22], the authors study finite-time passivity of multi-weighted coupled neural networks with and without coupling delays. As far as we know, very few scholars have discussed finite-time passivity of stochastic complex networks in recent years. 
Motivated by the above discussions, we will investigate finite-time passivity of stochastic coupled complex networks (SCCNs). The main novelty and contributions of this paper can be summarized as follows. Firstly, we give three concepts of finite-time passivity in the sense of expectation. Secondly, we develop several finite-time passivity criteria. Lastly, we establish the relationship between finite-time passivity and finite-time synchronization in the sense of expectation.

\section{Lemmas and Definitions}

In this section, we will give some lemmas and definitions.

\subsection{Lemmas}

Lemma 1 (see [23]). Assume that a continuous, positivedefinite function $W(t)$ satisfies the following differential inequality:

$$
\dot{W}(t) \leq-\varrho W^{\mu}(t), \quad t \geq t_{0}, W\left(t_{0}\right) \geq 0,
$$

where $\mathrm{\varrho}>0$ and $0<\mu<1$ are constants. Then, for any given $t_{0}$, $W(t)$ satisfies the following inequality:

$$
\begin{aligned}
W^{1-\mu}(t) & \leq W^{1-\mu}\left(t_{0}\right)-\varrho(1-\mu)\left(t-t_{0}\right), \quad t_{0} \leq t \leq t_{1}, \\
W(t) & \equiv 0, \quad t \geq t_{1},
\end{aligned}
$$

with $t_{1}$ given by

$$
t_{1}=t_{0}+\frac{W^{1-\mu}\left(t_{0}\right)}{\varrho(1-\mu)} .
$$

Lemma 2 (see [24]). For any $b_{i} \in \mathbb{R}, i=1, \ldots, n, 0<p \leq 1$, the following inequality holds:

$$
\left(\sum_{i=1}^{n}\left|b_{i}\right|\right)^{p} \leq \sum_{i=1}^{n}\left|b_{i}\right|^{p}
$$

Lemma 3 (see [25]). For any vectors $x, y \in \mathbb{R}^{n}$ and matrix $0<P \in \mathbb{R}^{n \times n}$, the following inequality holds:

$$
x^{T} y+y^{T} x \leq x^{T} P x+y^{T} P^{-1} y .
$$

2.2. Definitions. Next, we will give three definitions about finite-time passivity in the sense of expectation. $E\{\cdot\}$ in these definitions stands for the mathematical expectation operator with respect to the given probability.

Definition 1. A stochastic system with input $u(t) \in \mathbb{R}^{n}$ and output $y(t) \in \mathbb{R}^{n}$ is said to be finite-time passive in the sense of expectation if there exists a nonnegative function $V$ such that

$$
E\left\{u^{T}(t) y(t)\right\} \geq \frac{E\{\mathrm{~d} V(t)\}}{\mathrm{d} t}+\beta E\left\{V^{\alpha}(t)\right\}
$$

for some $\alpha \in(0,1)$ and $\beta>0$.
Definition 2. A stochastic system with input $u(t) \in \mathbb{R}^{n}$ and output $y(t) \in \mathbb{R}^{n}$ is finite-time input strictly passive in the sense of expectation if there exists a nonnegative function $V$ such that

$$
E\left\{u^{T}(t) y(t)\right\}-\gamma_{1} E\left\{u^{T}(t) u(t)\right\} \geq \frac{E\{\mathrm{~d} V(t)\}}{\mathrm{d} t}+\beta E\left\{V^{\alpha}(t)\right\},
$$

for some $\alpha \in(0,1), \beta>0$, and $\gamma_{1}>0$.

Definition 3. A stochastic system with input $u(t) \in \mathbb{R}^{n}$ and output $y(t) \in \mathbb{R}^{n}$ is finite-time output strictly passive in the sense of expectation if there exists a nonnegative function $V$ such that

$$
E\left\{u^{T}(t) y(t)\right\}-\gamma_{2} E\left\{y^{T}(t) y(t)\right\} \geq \frac{E\{\mathrm{~d} V(t)\}}{\mathrm{d} t}+\beta E\left\{V^{\alpha}(t)\right\},
$$

for some $\alpha \in(0,1), \beta>0$, and $\gamma_{2}>0$.

Definition 4 (see [25]). Let $A=\left(a_{i j}\right)_{m \times m} \in \mathbb{R}^{m \times n}$ and $B=\left(b_{i j}\right)_{p \times q} \in \mathbb{R}^{p \times q}$. Then, the Kronecker product of $A$ and $B$ is defined as the matrix

$$
A \otimes B=\left(\begin{array}{cccc}
a_{11} B & a_{12} B & \cdots & a_{2 n} B \\
a_{21} B & a_{22} B & \cdots & a_{2 n} B \\
\vdots & \vdots & \cdots & \vdots \\
a_{m 1} B & a_{m 2} B & \cdots & a_{m n} B
\end{array}\right) \in \mathbb{R}^{m p \times n q} .
$$

Throughout this paper, we make the following assumptions.

(H1) (see [26]) The function $f(\cdot)$ is in the QUAD class, that is, there exist diagonal matrices $0<P=\operatorname{diag}$ $\left(p_{1}, p_{2}, \ldots, p_{n}\right) \in \mathbb{R}^{n \times n}$ and $\Delta=\operatorname{diag}\left(\delta_{1}, \delta_{2}, \ldots, \delta_{n}\right)$ $\in \mathbb{R}^{n \times n}$, such that

$$
(x-y)^{T} P[f(x)-f(y)-\Delta(x-y)] \leq-\lambda(x-y)^{T}(x-y),
$$

for all $x, y \in \mathbb{R}^{n}$ and some $\lambda>0$.

(H2) For arbitrary $u, v \in \mathbb{R}^{n}$, there exists a positive constant $L$ such that the following inequality holds:

$$
\operatorname{trace}[h(u)-h(v)]^{T}[h(u)-h(v)] \leq L(u-v)^{T}(u-v) \text {. }
$$

Remark 1 (see [25]). It can be verified that many of the benchmark chaotic systems belong to "function class QUAD," such as the Lorenz system, the Chen system, and the $L \ddot{u}$ system.

\section{Finite-Time Passivity of SCCNs}

3.1. Network Model. In this paper, we will consider the following stochastic coupled complex networks model: 


$$
\begin{aligned}
\mathrm{d} z_{i}(t)= & {\left[f\left(z_{i}(t)\right)+a \sum_{j=1}^{N} G_{i j} \Gamma z_{j}(t)+u_{i}(t)+v_{i}(t)\right] \mathrm{d} t } \\
& +h\left(z_{i}(t)\right) \mathrm{d} \omega(t),
\end{aligned}
$$

where $z_{i}(t)=\left(z_{i 1}(t), z_{i 2}(t), \ldots, z_{i n}(t)\right)^{T} \in \mathbb{R}^{n}$ is the state vector of the $i$ th node; $N$ corresponds to the number of neurons; $f\left(z_{i}(t)\right)=\left(f_{1}\left(z_{i 1}(t)\right), f_{2}\left(z_{i 2}(t)\right), \ldots, f_{n}\left(z_{\text {in }}(t)\right)\right)^{T} \in \mathbb{R}^{n}$ denotes the neuron activation function and satisfies assumption (H1); $u_{i}(t)=\left(u_{i 1}(t), u_{i 2}(t), \ldots, u_{i n}(t)\right)^{T} \in \mathbb{R}^{n}$ is a varying external input vector; $v_{i}(t)=\left(v_{i 1}(t), v_{i 2}(t), \ldots, v_{i n}(t)\right)^{T} \in \mathbb{R}^{n}$ denotes the control input; $h(\cdot) \in \mathbb{R}^{n \times n}$ satisfies assumption $(\mathrm{H} 2) ; \quad \omega(t)=\left(\omega_{1}(t), \omega_{2}(t), \ldots, \omega_{n}(t)\right)^{T} \in \mathbb{R}^{n} \quad$ is a $n$-dimensional Brownian motion defined on a complete probability space $(\Omega, P) ; a$ is a positive real number which represents the overall coupling strength; $\Gamma$ denotes the inner coupling matrix; and $G=\left(G_{i j}\right)_{N \times N}$ represents the topological structure of the network, where $G_{i j}$ is defined as follows: if there exists a connection between node $i$ and node $j$, then $G_{i j}=G_{j i}>0$; otherwise, $G_{i j}=G_{j i}=0,(i \neq j)$, and the diagonal elements of matrix $G$ are defined by

$$
G_{i i}=-\sum_{\substack{j=1 \\ j \neq i}}^{N} G_{i j}, \quad i=1,2, \ldots, N .
$$

3.2. Finite-Time Passivity. Set synchronization function $\bar{z}(t)$ satisfies

$$
\mathrm{d} \bar{z}(t)=f(\bar{z}(t)) \mathrm{d} t+h(\bar{z}(t)) \mathrm{d} \omega(t),
$$

where $\bar{z}(t)=\left(\bar{z}_{1}(t), \bar{z}_{2}(t), \ldots, \bar{z}_{n}(t)\right) \in \mathbb{R}^{n}$.

Define $e_{i}(t)=z_{i}(t)-\bar{z}(t), i=1,2, \ldots, N$. Then, we have

$$
\begin{aligned}
\mathrm{d} e_{i}(t)= & {\left[f\left(z_{i}(t)\right)-f(\bar{z}(t))\right.} \\
& \left.+a \sum_{j=1}^{N} G_{i j} \Gamma e_{j}(t)+u_{i}(t)+v_{i}(t)\right] \mathrm{d} t \\
& +\left[h\left(z_{i}(t)\right)-h(\bar{z}(t))\right] \mathrm{d} \omega(t),
\end{aligned}
$$

where $i=1,2, \ldots, N$.

$y_{i}(t) \in \mathbb{R}^{n}$ refers to the output vector of (15) and is defined as follows:

$$
y_{i}(t)=A_{1} e_{i}(t)+A_{2} u_{i}(t),
$$

where $A_{1}, A_{2} \in \mathbb{R}^{n \times n}$,

The controller for network (12) is defined as follows:

$$
\begin{aligned}
v_{i}(t)= & -Q_{i}\left(z_{i}(t)-\bar{z}(t)\right) \\
& -\beta P^{((\alpha-1) / 2)} \operatorname{sign}\left(z_{i}(t)-\bar{z}(t)\right)\left|z_{i}(t)-\bar{z}(t)\right|^{\alpha},
\end{aligned}
$$

where $Q_{i} \in \mathbb{R}^{n \times n}, 0<\alpha<1, \beta>0, P$ is defined in (H1), and

$$
\begin{aligned}
\operatorname{sign}\left(z_{i}(t)-\bar{z}(t)\right) & =\operatorname{diag}\left(\operatorname{sign}\left(z_{i 1}(t)-\bar{z}_{1}(t)\right), \operatorname{sign}\left(z_{i 2}(t)-\bar{z}_{2}(t)\right), \ldots, \operatorname{sign}\left(z_{\text {in }}(t)-\bar{z}_{n}(t)\right)\right), \\
\left|z_{i}(t)-\bar{z}(t)\right|^{\alpha} & =\left(\left|z_{i 1}(t)-\bar{z}_{1}(t)\right|^{\alpha},\left|z_{i 2}(t)-\bar{z}_{2}(t)\right|^{\alpha}, \ldots,\left|z_{\text {in }}(t)-\bar{z}_{n}(t)\right|^{\alpha}\right)^{T}, \\
P^{((\alpha-1) / 2)} & =\operatorname{diag}\left(p_{1}^{((\alpha-1) / 2)}, p_{2}^{((\alpha-1) / 2)}, \ldots, p_{n}^{((\alpha-1) / 2)}\right) .
\end{aligned}
$$

Theorem 1. Under assumptions (H1) and (H2), network model (15) is finite-time passive in the sense of expectation under controller (17) if there exists matrix $Q=\operatorname{diag}\left(Q_{1}, Q_{2}, \ldots, Q_{N}\right) \in \mathbb{R}^{n N \times n N}$ such that

$$
\left(\begin{array}{cc}
K_{1} & E_{1} \\
E_{1}^{T} & -I_{N} \otimes \frac{A_{2}+A_{2}^{T}}{2}
\end{array}\right) \leq 0,
$$

where

$$
\begin{aligned}
K_{1}= & I_{N} \otimes\left(P \Delta+\Delta P-2 \lambda I_{n}+\lambda_{0} I_{n}\right)-\left(I_{N} \otimes P\right) Q \\
& -Q^{T}\left(I_{N} \otimes P\right)+a G \otimes(P \Gamma+\Gamma P), \\
E_{1}= & I_{N} \otimes P-\frac{I_{N} \otimes A_{1}^{T}}{2} .
\end{aligned}
$$

According to Ito's lemma, we acquire from (15) and (17)

$$
\mathrm{d} V_{1}(t)=\mathscr{L} V_{1}(t) \mathrm{d} t+\Phi(t) \mathrm{d} \omega(t)
$$

Here

$$
\begin{aligned}
\mathscr{L} V_{1}(t)= & 2 \sum_{i=1}^{N} e_{i}^{T}(t) P\left[f\left(z_{i}(t)\right)-f(\bar{z}(t))+a \sum_{j=1}^{N} G_{i j} \Gamma e_{j}(t)\right. \\
& \left.+u_{i}(t)-Q_{i} e_{i}(t)-\beta P^{((\alpha-1) / 2)} \operatorname{sign}\left(e_{i}(t)\right)\left|e_{i}(t)\right|^{\alpha}\right] \\
& +\sum_{i=1}^{N} \operatorname{trace}\left[h\left(z_{i}(t)\right)-h(\bar{z}(t))\right]^{T} P\left[h\left(z_{i}(t)\right)-h(\bar{z}(t))\right], \\
\Phi(t)= & 2 \sum_{i=1}^{N} e_{i}^{T}(t) P\left[h\left(z_{i}(t)\right)-h(\bar{z}(t))\right] .
\end{aligned}
$$

Proof. For network (15), the Lyapunov functional is chosen as follows:

According to (H1), we can obtain 


$$
\sum_{i=1}^{N} e_{i}^{T}(t) P\left(f\left(z_{i}(t)\right)-f(\bar{z}(t))\right) \leq \sum_{i=1}^{N} e_{i}^{T}(t)\left(P \Delta-\lambda I_{n}\right) e_{i}(t) .
$$

We can get the following from $(\mathrm{H} 2)$ :

$$
\begin{aligned}
& \operatorname{trace}\left[h\left(z_{i}(t)\right)-h(\bar{z}(t))\right]^{T} P\left[h\left(z_{i}(t)\right)-h(\bar{z}(t))\right] \\
& \quad \leq \lambda_{M}(P) \operatorname{trace}\left[h\left(z_{i}(t)\right)-h(\bar{z}(t))\right]^{T}\left[h\left(z_{i}(t)\right)-h(\bar{z}(t))\right] \\
& \quad \leq L \lambda_{M}(P)\left[z_{i}(t)-\bar{z}(t)\right]^{T}\left[z_{i}(t)-\bar{z}(t)\right]=\lambda_{0} e_{i}^{T}(t) e_{i}(t) .
\end{aligned}
$$

Here $\lambda_{M}(P)$ represents maximum eigenvalue of matrix $P, \lambda_{0}=L \lambda_{M}(P)$.

Thus,

$$
\begin{aligned}
\mathscr{L} V_{1}(t) \leq & \sum_{i=1}^{N} e_{i}^{T}(t)\left(P \Delta+\Delta P-2 \lambda I_{n}+\lambda_{0} I_{n}\right) e_{i}(t) \\
& +2 a \sum_{i=1}^{N} \sum_{j=1}^{N} G_{i j} e_{i}^{T}(t) P \Gamma e_{j}(t) \\
& +2 \sum_{i=1}^{N} e_{i}^{T}(t) P u_{i}(t)-2 \sum_{i=1}^{N} e_{i}^{T}(t) P Q_{i} e_{i}(t) \\
& -2 \beta \sum_{i=1}^{N} e_{i}^{T}(t) P^{((\alpha+1) / 2)} \operatorname{sign}\left(e_{i}(t)\right)\left|e_{i}(t)\right|^{\alpha} \\
= & e^{T}(t)\left[I_{N} \otimes\left(P \Delta+\Delta P-2 \lambda I_{n}+\lambda_{0} I_{n}\right)\right. \\
& -\left(I_{N} \otimes P\right) Q-Q^{T}\left(I_{N} \otimes P\right) \\
& +a G \otimes(P \Gamma+\Gamma P)] e(t)+2 e^{T}(t)\left(I_{N} \otimes P\right) u(t) \\
& -2 \beta \sum_{i=1}^{N} e_{i}^{T}(t) P^{((\alpha+1) / 2)} \operatorname{sign}\left(e_{i}(t)\right)\left|e_{i}(t)\right|^{\alpha},
\end{aligned}
$$

where $e(t)=\left(e_{1}^{T}(t), e_{2}^{T}(t), \ldots, e_{N}^{T}(t)\right)^{T}, \quad u(t)=\left(u_{1}^{T}(t)\right.$, $\left.u_{2}^{T}(t), \ldots, u_{N}^{T}(t)\right)^{T}$.

Considering $\operatorname{sign} x \cdot x=|x|,(\forall x \in \mathbb{R})$ and Lemma 2 , we can easily conclude that

$$
\begin{gathered}
\sum_{i=1}^{N} e_{i}^{T}(t) P^{((\alpha+1) / 2)} \operatorname{sign}\left(e_{i}(t)\right)\left|e_{i}(t)\right|^{\alpha} \\
=\sum_{i=1}^{N} \sum_{j=1}^{n} p_{j}^{((\alpha+1) / 2)}\left|e_{i j}(t)\right|^{\alpha+1} \\
\geq \sum_{i=1}^{N}\left(\sum_{j=1}^{n} p_{j} e_{i j}^{2}(t)\right)^{((\alpha+1) / 2)} \\
=\sum_{i=1}^{N}\left(e_{i}^{T}(t) P e_{i}(t)\right)^{((\alpha+1) / 2)}
\end{gathered}
$$

Set $y(t)=\left(y_{1}^{T}(t), y_{2}^{T}(t), \ldots, y_{N}^{T}(t)\right)^{T}$; consequently,

$$
\begin{aligned}
u^{T}(t) y(t)= & \sum_{i=1}^{N} u_{i}^{T}(t) y_{i}(t) \\
= & \sum_{i=1}^{N} u_{i}^{T}(t)\left[A_{1} e_{i}(t)+A_{2} u_{i}(t)\right] \\
= & u^{T}(t)\left(I_{N} \otimes A_{1}\right) e(t) \\
& +u^{T}(t)\left(I_{N} \otimes A_{2}\right) u(t) .
\end{aligned}
$$

From (19) and (26)-(28),

$$
\begin{aligned}
& \mathscr{L} V_{1}(t)-u^{T}(t) y(t) \\
& \leq-2 \beta \sum_{i=1}^{N}\left(e_{i}^{T}(t) P e_{i}(t)\right)^{((\alpha+1) / 2)} \\
& +\zeta^{T}(t)\left(\begin{array}{cc}
K_{1} & E_{1} \\
E_{1}^{T} & -I_{N} \otimes \frac{A_{2}+A_{2}^{T}}{2}
\end{array}\right) \zeta(t) \\
& \leq-2 \beta \sum_{i=1}^{N}\left(e_{i}^{T}(t) P e_{i}(t)\right)^{((\alpha+1) / 2)} \\
& \leq-2 \beta\left(\sum_{i=1}^{N} e_{i}^{T}(t) P e_{i}(t)\right)^{((\alpha+1) / 2)} \\
& =-2 \beta V_{1}^{((\alpha+1) / 2)}(t),
\end{aligned}
$$

where $\zeta(t)=\left(e^{T}(t), u^{T}(t)\right)^{T}$.

Considering $E\left\{\mathrm{~d} V_{1}(t)\right\}=E\left\{\mathscr{L} V_{1}(t) \mathrm{d} t\right\}$, consequently

$$
\begin{aligned}
E\{ & \left.\mathrm{d} V_{1}(t)-u^{T}(t) y(t) \mathrm{d} t\right\} \\
& =E\left\{\left[\mathscr{L} V_{1}(t)-u^{T}(t) y(t)\right] \mathrm{d} t\right\} \\
& \leq-2 \beta E\left\{V_{1}^{((\alpha+1) / 2)}(t) \mathrm{d} t\right\} .
\end{aligned}
$$

Then, we can obtain

$$
E\left\{u^{T}(t) y(t)\right\} \geq \frac{E\left\{\mathrm{~d} V_{1}(t)\right\}}{\mathrm{d} t}+2 \beta E\left\{V_{1}^{((\alpha+1) / 2)}(t)\right\} .
$$

Therefore, network (15) is finite-time passive in the sense of expectation under controller (17).

Theorem 2. Under assumptions (H1) and (H2), network model (15) is finite-time input strictly passive in the sense of expectation under controller (17) if there exist matrix $Q=$ $\operatorname{diag}\left(Q_{1}, Q_{2}, \ldots, Q_{N}\right) \in \mathbb{R}^{n N \times n N}$ and a positive real number $\gamma_{1}$ such that

$$
\left(\begin{array}{cc}
K_{1} & E_{1} \\
E_{1}^{T} & \gamma_{1} I_{n N}-I_{N} \otimes \frac{A_{2}+A_{2}^{T}}{2}
\end{array}\right) \leq 0,
$$


where $K_{1}, E_{1}$ have the same meanings as in Theorem 1.

Proof. We will choose the same $V_{1}(t)$ as (21) for network (15).

By (26)-(28), one can get

$$
\begin{aligned}
& \mathscr{L} V_{1}(t)-u^{T}(t) y(t)+\gamma_{1} u^{T}(t) u(t) \\
& \leq e^{T}(t)\left[I_{N} \otimes\left(P \Delta+\Delta P-2 \lambda I_{n}+\lambda_{0} I_{n}\right)\right. \\
& -\left(I_{N} \otimes P\right) Q-Q^{T}\left(I_{N} \otimes P\right) \\
& +a G \otimes(P \Gamma+\Gamma P)] e(t) \\
& +2 e^{T}(t)\left(I_{N} \otimes P\right) u(t)+\gamma_{1} u^{T}(t) u(t) \\
& -2 \beta \sum_{i=1}^{N} e_{i}^{T}(t) P^{((\alpha+1) / 2)} \operatorname{sign}\left(e_{i}(t)\right)\left|e_{i}(t)\right|^{\alpha} \\
& -u^{T}(t)\left(I_{N} \otimes A_{1}\right) e(t)-u^{T}(t)\left(I_{N} \otimes A_{2}\right) u(t) \\
& =-2 \beta \sum_{i=1}^{N} e_{i}^{T}(t) P^{((\alpha+1) / 2)} \operatorname{sign}\left(e_{i}(t)\right)\left|e_{i}(t)\right|^{\alpha} \\
& +\zeta^{T}(t)\left(\begin{array}{cc}
K_{1} & E_{1} \\
E_{1}^{T} & \gamma_{1} I_{n N}-I_{N} \otimes \frac{A_{2}+A_{2}^{T}}{2}
\end{array}\right) \zeta(t) \\
& \leq-2 \beta\left(\sum_{i=1}^{N} e_{i}^{T}(t) P e_{i}(t)\right)^{((\alpha+1) / 2)}=-2 \beta V_{1}^{((\alpha+1) / 2)}(t) .
\end{aligned}
$$

Taking the mathematical expectation on both sides above, one can derive that

$$
\begin{aligned}
& E\left\{u^{T}(t) y(t)\right\}-\gamma_{1} E\left\{u^{T}(t) u(t)\right\} \\
& \geq \frac{E\left\{\mathrm{~d} V_{1}(t)\right\}}{\mathrm{d} t}+2 \beta E\left\{V_{1}^{((\alpha+1) / 2)}(t)\right\} .
\end{aligned}
$$

Therefore, network (15) is finite-time input strictly passive in the sense of expectation under controller (17).

Theorem 3. Under assumptions (H1) and (H2), network model (15) is finite-time output strictly passive in the sense of expectation under controller (17) if there exist matrix $Q=$ $\operatorname{diag}\left(Q_{1}, Q_{2}, \ldots, Q_{N}\right) \in \mathbb{R}^{n N \times n N}$ and a positive real number $\gamma_{2}$ such that

$$
\left(\begin{array}{ll}
K_{2} & E_{2} \\
E_{2}^{T} & K_{3}
\end{array}\right) \leq 0
$$

where

$$
\begin{aligned}
& K_{2}=K_{1}+\gamma_{2}\left(I_{N} \otimes A_{1}^{T}\right)\left(I_{N} \otimes A_{1}\right), \\
& E_{2}=E_{1}+\gamma_{2}\left(I_{N} \otimes A_{1}^{T}\right)\left(I_{N} \otimes A_{2}\right), \\
& K_{3}=\gamma_{2}\left(I_{N} \otimes A_{2}^{T}\right)\left(I_{N} \otimes A_{2}\right)-I_{N} \otimes \frac{A_{2}+A_{2}^{T}}{2},
\end{aligned}
$$

Proof. Firstly we calculate the following equality:

$$
\begin{aligned}
y^{T}(t) y(t)= & \sum_{i=1}^{N} y_{i}^{T}(t) y_{i}^{T}(t) \\
= & \sum_{i=1}^{N}\left[A_{1} e_{i}(t)+A_{2} u_{i}(t)\right]^{T}\left[A_{1} e_{i}(t)+A_{2} u_{i}(t)\right] \\
= & \sum_{i=1}^{N}\left[e_{i}^{T}(t) A_{1}^{T}+u_{i}^{T}(t) A_{2}^{T}\right]\left[A_{1} e_{i}(t)+A_{2} u_{i}(t)\right] \\
= & \sum_{i=1}^{N}\left[e_{i}^{T}(t) A_{1}^{T} A_{1} e_{i}(t)+e_{i}^{T}(t) A_{1}^{T} A_{2} u_{i}(t)\right. \\
& \left.+u_{i}^{T}(t) A_{2}^{T} A_{1} e_{i}(t)+u_{i}^{T}(t) A_{2}^{T} A_{2} u_{i}(t)\right] \\
= & e^{T}(t)\left(I_{N} \otimes A_{1}^{T} A_{1}\right) e(t)+e^{T}(t)\left(I_{N} \otimes A_{1}^{T} A_{2}\right) u(t) \\
& +u^{T}(t)\left(I_{N} \otimes A_{2}^{T} A_{1}\right) e(t)+u^{T}(t)\left(I_{N} \otimes A_{2}^{T} A_{2}\right) u(t) \\
= & e^{T}(t)\left(I_{N} \otimes A_{1}^{T}\right)\left(I_{N} \otimes A_{1}\right) e(t) \\
& +e^{T}(t)\left(I_{N} \otimes A_{1}^{T}\right)\left(I_{N} \otimes A_{2}\right) u(t) \\
& +u^{T}(t)\left(I_{N} \otimes A_{2}^{T}\right)\left(I_{N} \otimes A_{1}\right) e(t) \\
& +u^{T}(t)\left(I_{N} \otimes A_{2}^{T}\right)\left(I_{N} \otimes A_{2}\right) u(t) .
\end{aligned}
$$

For the last step, we utilize the important properties of the Kronecker product:

$$
\left(M_{1} \otimes M_{2}\right)\left(M_{3} \otimes M_{4}\right)=\left(M_{1} M_{3}\right) \otimes\left(M_{2} M_{4}\right) .
$$

Select the same $V_{1}(t)$ as (21) for network (15). We can obtain

$$
\begin{aligned}
\mathscr{L} V_{1} & (t)-u^{T}(t) y(t)+\gamma_{2} y^{T}(t) y(t) \\
\leq & e^{T}(t)\left[I_{N} \otimes\left(P \Delta+\Delta P-2 \lambda I_{n}+\lambda_{0} I_{n}\right)\right. \\
& -\left(I_{N} \otimes P\right) Q-Q^{T}\left(I_{N} \otimes P\right) \\
& +a G \otimes(P \Gamma+\Gamma P)] e(t)+2 e^{T}(t)\left(I_{N} \otimes P\right) u(t) \\
& -u^{T}(t)\left(I_{N} \otimes A_{1}\right) e(t)-u^{T}(t)\left(I_{N} \otimes A_{2}\right) u(t) \\
& +\gamma_{2} e^{T}(t)\left(I_{N} \otimes A_{1}^{T}\right)\left(I_{N} \otimes A_{1}\right) e(t) \\
& +\gamma_{2} e^{T}(t)\left(I_{N} \otimes A_{1}^{T}\right)\left(I_{N} \otimes A_{2}\right) u(t) \\
& +\gamma_{2} u^{T}(t)\left(I_{N} \otimes A_{2}^{T}\right)\left(I_{N} \otimes A_{1}\right) e(t) \\
& +\gamma_{2} u^{T}(t)\left(I_{N} \otimes A_{2}^{T}\right)\left(I_{N} \otimes A_{2}\right) u(t) \\
& -2 \beta \sum_{i=1}^{N} e_{i}^{T}(t) P^{((\alpha+1) / 2)} \operatorname{sign}\left(e_{i}(t)\right)\left|e_{i}(t)\right|^{\alpha} \\
= & -2 \beta \sum_{i=1}^{N} e_{i}^{T}(t) P^{((\alpha+1) / 2)} \operatorname{sign}\left(e_{i}(t)\right)\left|e_{i}(t)\right|^{\alpha} \\
& +\zeta^{T}(t)\left(\begin{array}{l}
K_{2} \\
E_{2}^{T} E_{2} \\
K_{3}
\end{array}\right) \zeta(t) \\
& -2 \beta\left(\sum_{i=1}^{N} e_{i}^{T}(t) P e_{i}(t)\right)^{((\alpha+1) / 2)}=-2 \beta V_{1}^{((\alpha+1) / 2)}(t) .
\end{aligned}
$$

$K_{1}, E_{1}$ have the same meanings as in Theorem 1. 
Taking the mathematical expectation on both sides above, one can derive that

$$
\begin{aligned}
E\left\{u^{T}(t) y(t)\right\}-\gamma_{2} E\left\{y^{T}(t) y(t)\right\} \\
\geq \frac{E\left\{\mathrm{~d} V_{1}(t)\right\}}{\mathrm{d} t}+2 \beta E\left\{V_{1}^{((\alpha+1) / 2)}(t)\right\} .
\end{aligned}
$$

Therefore, network (15) is finite-time output strictly passive in the sense of expectation under controller (17).

3.3. Finite-Time Synchronization. In this section, we will verify finite-time synchronization in the sense of expectation for SCCNs (12). Firstly, the definition of finite-time synchronization is given as follows.

Definition 5. The SCCN (12) is finite-time synchronized in the sense of expectation if there exists a constant $T>0$ such that

$$
\begin{aligned}
\lim _{t \longrightarrow T^{-}} E\left\{\left\|z_{i}(t)-\bar{z}(t)\right\|_{2}\right\} & =0, \\
E\left\{\left\|z_{i}(t)-\bar{z}(t)\right\|_{2}\right\} & \equiv 0, \quad t \geq T,
\end{aligned}
$$

for $i=1,2, \ldots, N$, where $u_{i}(t)=0, i=1,2, \ldots, N$.

Theorem 4. Assume that a continuous, positive-definite function $V(t)$ satisfies the following inequality:

$$
\varphi_{1}\left(E\|e(t)\|_{2}\right) \leq V(t),
$$

where $\varphi_{1}:[0,+\infty) \longrightarrow[0,+\infty)$ is continuous and strictly monotonically increasing function and $\varphi_{1}(s)$ is positive for $s>0$ with $\varphi_{1}(0)=0$. If network (15) is finite-time passive (finite-time input strictly passive, finite-time output strictly passive) in the sense of expectation with respect to $V(t)$, then SCCN (12) is finite-time synchronized in the sense of expectation under controller (17).

Proof. The network model (15) is finite-time passive in the sense of expectation with respect to $V(t)$ under controller (17), that is to say, there exist $\alpha \in(0,1)$ and $\beta>0$ such that

$$
E\left\{u^{T}(t) y(t)\right\} \geq \frac{E\{\mathrm{~d} V(t)\}}{\mathrm{d} t}+\beta E\left\{V^{\alpha}(t)\right\}
$$

Considering $u(t)=0$, one obtains

$$
\frac{E\{\mathrm{~d} V(t)\}}{\mathrm{d} t}+\beta E\left\{V^{\alpha}(t)\right\} \leq 0 .
$$

According to the property of mathematical expectation,

$$
\dot{V}(t) \leq-\beta V^{\alpha}(t) \text {. }
$$

Choosing $t_{0}=0$ in Lemma 1 , we can obtain $V(t) \equiv 0$ for $t \geqslant t_{1}$, where $t_{1}=\left(V^{1-\alpha}(0) / \beta(1-\alpha)\right)$. On the one hand, since

$$
\varphi_{1}\left[E\left(\|e(t)\|_{2}\right)\right] \leq V(t)
$$

one has

$$
\varphi_{1}\left[E\left(\|e(t)\|_{2}\right)\right] \leq V(t) \equiv 0,
$$

for $t \geq t_{1}$. Since $\varphi_{1}(s)=0$ if and only if $s=0$. Then, we can conclude that

$$
E\left(\|e(t)\|_{2}\right) \equiv 0, \quad t \geq t_{1} .
$$

On the other hand, $V(t)$ is continuous, so

$$
\lim _{t \longrightarrow t_{1}^{-}} V(t)=\lim _{t \longrightarrow t_{1}^{+}} V(t)=0 .
$$

Taking the limit $t \longrightarrow t_{1}^{-}$on both sides of (46), we will get

$$
\lim _{t \longrightarrow t_{1}^{-}} \varphi_{1}\left[E\left(\|e(t)\|_{2}\right)\right]=0 .
$$

Namely, SCCN (12) is finite-time synchronized in the sense of expectation under controller (17).

Similarly, it is easy to prove that SCCN (12) is also finitetime synchronized in the sense of expectation under controller (17) if network model (15) is finite-time input strictly passive or finite-time output strictly passive in the sense of expectation.

\section{Finite-Time Passivity of SCCNs with Time- Varying Delay}

4.1. Network Model. In this section, the network model is described by

$$
\begin{aligned}
\mathrm{d} z_{i}(t)= & {\left[f\left(z_{i}(t)\right)+a \sum_{j=1}^{N} G_{i j} \Gamma z_{j}(t-\tau(t))\right.} \\
& \left.+u_{i}(t)+v_{i}(t)\right] \mathrm{d} t+h\left(z_{i}(t)\right) \mathrm{d} \omega(t),
\end{aligned}
$$

where $\tau(t)$ is the time delay and satisfies $0 \leq \tau(t) \leq$ $\tau, 0 \leq \dot{\tau}(t) \leq d<1$.

4.2. Finite-Time Passivity. Let $\bar{z}(t)$ also satisfy

$$
\mathrm{d} \bar{z}(t)=f(\bar{z}(t)) \mathrm{d} t+h(\bar{z}(t)) \mathrm{d} \omega(t) .
$$

Define $e_{i}(t)=z_{i}(t)-\bar{z}(t), i=1,2, \ldots, N$. Then, we have

$$
\begin{aligned}
\mathrm{d} e_{i}(t)= & {\left[f\left(z_{i}(t)\right)-f(\bar{z}(t))\right.} \\
& \left.+a \sum_{j=1}^{N} G_{i j} \Gamma e_{j}(t-\tau(t))+u_{i}(t)+v_{i}(t)\right] \mathrm{d} t \\
& +\left[h\left(z_{i}(t)\right)-h(\bar{z}(t))\right] \mathrm{d} \omega(t) .
\end{aligned}
$$
follows:

$$
y_{i}(t)=B_{1} e_{i}(t)+B_{2} u_{i}(t)
$$

Design the following controller for network (51): 


$$
\begin{aligned}
v_{i}(t)= & -\beta P^{-1}\left(\frac { a } { 1 - d } \int _ { t - \tau ( t ) } ^ { t } ( z _ { i } ( h ) - \overline { z } ( h ) ) ^ { T } M _ { i } \left(z_{i}(h)\right.\right. \\
& -\bar{z}(h)) \mathrm{d} h)^{((\alpha+1) / 2)} \frac{z_{i}(t)-\bar{z}(t)}{\left\|z_{i}(t)-\bar{z}(t)\right\|_{2}^{2}} \\
& -\beta P^{((\alpha-1) / 2)} \operatorname{sign}\left(z_{i}(t)-\bar{z}(t)\right)\left|z_{i}(t)-\bar{z}(t)\right|^{\alpha} \\
& -Q_{i}\left(z_{i}(t)-\bar{z}(t)\right)
\end{aligned}
$$

where $\quad 0<M_{i} \in \mathbb{R}^{n \times n}, M=\operatorname{diag}\left(M_{1}, M_{2}, \ldots, M_{N}\right), Q_{i}, \alpha$, $P, \beta, P^{((\alpha-1) / 2)}$, sign $\left(z_{i}(t)-\bar{z}(t)\right),\left|z_{i}(t)-\bar{z}(t)\right|^{\alpha}$ have the same meanings as in (17).

Theorem 5. Under assumptions (H1) and (H2), network model (53) is finite-time passive in the sense of expectation under controller (55) if there exist matrices $Q=\operatorname{diag}\left(Q_{1}\right.$, $\left.Q_{2}, \ldots, Q_{N}\right) \in \mathbb{R}^{n N \times n N}, 0<M=\operatorname{diag}\left(M_{1}, M_{2}, \ldots, M_{N}\right) \in$ $\mathbb{R}^{n N \times n N}$ such that

$$
\left(\begin{array}{cc}
W_{1} & \Omega_{1} \\
\Omega_{1}^{T} & -I_{N} \otimes \frac{B_{2}+B_{2}^{T}}{2}
\end{array}\right) \leq 0,
$$

where

$$
\begin{aligned}
W_{1}= & I_{N} \otimes\left(P \Delta+\Delta P-2 \lambda I_{n}+\lambda_{0} I_{n}\right) \\
& -\left(I_{N} \otimes P\right) Q-Q^{T}\left(I_{N} \otimes P\right)+\frac{a}{1-d} M \\
& +a[G \otimes(P \Gamma)] M^{-1}[G \otimes(\Gamma P)], \\
& \Omega_{1}=I_{N} \otimes P-\frac{I_{N} \otimes B_{1}^{T}}{2} .
\end{aligned}
$$

Proof. Choose the following Lyapunov functional for network (51):

$$
\begin{aligned}
V_{2}(t)= & \sum_{i=1}^{N} e_{i}^{T}(t) P e_{i}(t) \\
& +\frac{a}{1-d} \int_{t-\tau(t)}^{t} e^{T}(h) M e(h) \mathrm{d} h,
\end{aligned}
$$

where $e(t)=\left(e_{1}^{T}(t), e_{2}^{T}(t), \ldots, e_{N}^{T}(t)\right)^{T}$.

According to Ito's lemma, we acquire from (53) and (55)

$$
\mathrm{d} V_{2}(t)=\mathscr{L} V_{2}(t) \mathrm{d} t+\Phi(t) \mathrm{d} \omega(t)
$$

Here

$$
\begin{aligned}
\mathscr{L} V_{2}(t)= & 2 \sum_{i=1}^{N} e_{i}^{T}(t) P\left[f\left(z_{i}(t)\right)-f(\bar{z}(t))+a \sum_{j=1}^{N} G_{i j} \Gamma e_{j}(t-\tau(t))\right. \\
& \left.+u_{i}(t)-Q_{i} e_{i}(t)-\beta P^{((\alpha-1) / 2)} \operatorname{sign}\left(e_{i}(t)\right)\left|e_{i}(t)\right|^{\alpha}\right] \\
& +\sum_{i=1}^{N} \operatorname{trace}\left[h\left(z_{i}(t)\right)-h(\bar{z}(t))\right]^{T} P\left[h\left(z_{i}(t)\right)-h(\bar{z}(t))\right] \\
& -2 \beta \sum_{i=1}^{N}\left(\frac{a}{1-d} \int_{t-\tau(t)}^{t} e_{i}^{T}(h) M_{i} e_{i}(h) \mathrm{d} h\right)^{((\alpha+1) / 2)} \\
& -2 \beta \sum_{i=1}^{N} e_{i}^{T}(t) P^{((\alpha+1) / 2)} \operatorname{sign}\left(e_{i}(t)\right)\left|e_{i}(t)\right|^{\alpha} \\
& +\frac{a}{1-d}\left[e^{T}(t) M e(t)\right. \\
& \left.-e^{T}(t-\tau(t)) M e(t-\tau(t))(1-\dot{\tau}(t))\right] \\
\Phi(t)= & 2 \sum_{i=1}^{N} e_{i}^{T}(t) P\left[h\left(z_{i}(t)\right)-h(\bar{z}(t))\right] .
\end{aligned}
$$

According to Lemma 3, we can take

$$
\begin{aligned}
& x=e(t-\tau(t)), \\
& y=[G \otimes(\Gamma P)] e(t) .
\end{aligned}
$$

It is not difficult to obtain

$$
\begin{aligned}
& 2 a e^{T}(t) {[G \otimes(P \Gamma)] e(t-\tau(t)) } \\
& \leq a e^{T}(t)[G \otimes(P \Gamma)] M^{-1}[G \otimes(\Gamma P)] e(t) \\
&+a e^{T}(t-\tau(t)) M e(t-\tau(t)) .
\end{aligned}
$$

From the above, one has

$$
\begin{aligned}
\mathscr{L} V_{2}(t) \leq & e^{T}(t)\left[I_{N} \otimes\left(P \Delta+\Delta P-2 \lambda I_{n}+\lambda_{0} I_{n}\right)\right. \\
& \left.-\left(I_{N} \otimes P\right) Q-Q^{T}\left(I_{N} \otimes P\right)\right] e(t) \\
& +2 a e^{T}(t)[G \otimes(P \Gamma)] e(t-\tau(t)) \\
& -2 \beta\left(\frac{a}{1-d} \int_{t-\tau(t)}^{t} e^{T}(h) M e(h) \mathrm{d} h\right)^{((\alpha+1) / 2)} \\
& -2 \beta\left(\sum_{i=1}^{N} e_{i}^{T}(t) P e_{i}(t)\right) \\
& -a e^{T}(t-\tau(t)) M e(t-\tau(t)) \\
& +\frac{a}{1-d} e^{T}(t) M e(t)+2 e^{T}(t)\left(I_{N} \otimes P\right) u(t)
\end{aligned}
$$




$$
\begin{aligned}
& \leq e^{T}(t)\left[I_{N} \otimes\left(P \Delta+\Delta P-2 \lambda I_{n}+\lambda_{0} I_{n}\right)\right. \\
& -\left(I_{N} \otimes P\right) Q-Q^{T}\left(I_{N} \otimes P\right)+\frac{a}{1-d} M \\
& \left.+a[G \otimes(P \Gamma)] M^{-1}[G \otimes(\Gamma P)]\right] e(t) \\
& -2 \beta\left(\frac{a}{1-d} \int_{t-\tau(t)}^{t} e^{T}(h) M e(h) \mathrm{d} h\right)^{((\alpha+1) / 2)} \\
& -2 \beta\left(\sum_{i=1}^{N} e_{i}^{T}(t) P e_{i}(t)\right)^{((\alpha+1) / 2)} \\
& +2 e^{T}(t)\left(I_{N} \otimes P\right) u(t) .
\end{aligned}
$$

Thus,

$$
\begin{aligned}
& \mathscr{L} V_{2}(t)-u^{T}(t) y(t) \\
\leq & \zeta^{T}(t)\left(\begin{array}{c}
W_{1} \quad \Omega_{1} \\
\Omega_{1}^{T}-I_{N} \otimes \frac{B_{2}+B_{2}^{T}}{2}
\end{array}\right) \zeta(t) \\
& -2 \beta\left(\frac{a}{1-d} \int_{t-\tau(t)}^{t} e^{T}(h) M e(h) \mathrm{d} h\right)^{((\alpha+1) / 2)} \\
& -2 \beta\left(\sum_{i=1}^{N} e_{i}^{T}(t) P e_{i}(t)\right)^{((\alpha+1) / 2)} \\
\leq & -2 \beta\left\{\left(\frac{a}{1-d} \int_{t-\tau(t)}^{t} e^{T}(h) M e(h) \mathrm{d} h\right)^{((\alpha+1) / 2)}\right. \\
& \left.\left.+\frac{a}{1-d} \int_{t-\tau(t)}^{t} e^{T}(h) M e(h) \mathrm{d} h\right)^{((\alpha+1) / 2)} e_{i}^{T}(t) P e_{i}(t)\right)^{((\alpha+1) / 2)} \\
& +2 \beta\left(\sum_{i=1}^{N} e_{i}^{T}(t) P e_{i}(t)\right. \\
\leq & -2 \beta+1) / 2)(t),
\end{aligned}
$$

where $u(t)=\left(u_{1}^{T}(t), u_{2}^{T}(t), \ldots, u_{N}^{T}(t)\right)^{T}, y(t)=\left(y_{1}^{T}(t)\right.$, $\left.y_{2}^{T}(t), \ldots, y_{N}^{T}(t)\right)^{T}, \zeta(t)=\left(e^{T}(t), u^{T}(t)\right)^{T}$.

Taking the mathematical expectation on (59), we can obtain

$$
E\left\{u^{T}(t) y(t)\right\} \geq \frac{E\left\{\mathrm{~d} V_{2}(t)\right\}}{\mathrm{d} t}+2 \beta E\left\{V_{2}^{((\alpha+1) / 2)}(t)\right\} .
$$

Consequently, network model (53) is finite-time passive in the sense of expectation under controller (55).

Theorem 6. Under assumptions (H1) and (H2), network model (53) is finite-time input strictly passive in the sense of expectation under controller (55) if there exist matrices $Q=$ $\operatorname{diag}\left(Q_{1}, Q_{2}, \ldots, Q_{N}\right) \in \mathbb{R}^{n N \times n N}, \quad 0<M=\operatorname{diag}\left(M_{1}, M_{2}\right.$, $\left.\ldots, M_{N}\right) \in \mathbb{R}^{n N \times n N}$ and a positive real number $\gamma_{3}$ such that

$$
\left(\begin{array}{cc}
W_{1} & \Omega_{1} \\
\Omega_{1}^{T} & \gamma_{3} I_{n N}-I_{N} \otimes \frac{B_{2}+B_{2}^{T}}{2}
\end{array}\right) \leq 0,
$$

where $W_{1}, \Omega_{1}$ have the same meanings as in Theorem 5.

Proof. We also select the same $V_{2}(t)$ as (58) for network (53). By (64), we get

$$
\begin{aligned}
\mathscr{L} V_{2}(t)- & u^{T}(t) y(t)+\gamma_{3} u^{T}(t) u(t) \\
\leq & \zeta^{T}(t)\left(\begin{array}{cc}
W_{1} \quad \Omega_{1} \\
\Omega_{1}^{T} \quad \gamma_{3} I_{n N}-I_{N} \otimes \frac{A_{2}+A_{2}^{T}}{2}
\end{array}\right) \zeta(t) \\
& -2 \beta\left\{\left(\frac{a}{1-d} \int_{t-\tau(t)}^{t} e^{T}(h) M e(h) \mathrm{d} h\right)^{((\alpha+1) / 2)}\right. \\
& +\left(\sum_{i=1}^{N} e_{i}^{T}(t) P e_{i}(t)\right)^{((\alpha+1) / 2)} \\
\leq & -2 \beta\left(\frac{a}{1-d} \int_{t-\tau(t)}^{t} e^{T}(h) M e(h) \mathrm{d} h\right. \\
& \left.+\sum_{i=1}^{N} e_{i}^{T}(t) P e_{i}(t)\right)^{((\alpha+1) / 2)}=-2 \beta V_{2}^{((\alpha+1) / 2)}(t) .
\end{aligned}
$$

Taking the mathematical expectation on (59), we can obtain

$$
\begin{aligned}
& E\left\{u^{T}(t) y(t)\right\}-\gamma_{3} E\left\{u^{T}(t) u(t)\right\} \\
\geq & \frac{E\left\{d V_{2}(t)\right\}}{d t}+2 \beta E\left\{V_{2}^{((\alpha+1) / 2)}(t)\right\} .
\end{aligned}
$$

Therefore, network (53) is finite-time input strictly passive in the sense of expectation under controller (55).

Theorem 7. Under assumptions (H1) and (H2), network model (53) is finite-time output strictly passive in the sense of expectation under controller (55) if there exist matrices $Q=\operatorname{diag}\left(Q_{1}, Q_{2}\right.$, $\left.\ldots, Q_{N}\right) \in \mathbb{R}^{n N \times n N}, \quad 0<M=\operatorname{diag}\left(M_{1}, M_{2}, \ldots, M_{N}\right) \in$ $\mathbb{R}^{n N \times n N}$ and a positive real number $\gamma_{4}$ such that 


$$
\left(\begin{array}{ll}
W_{2} & \Omega_{2} \\
\Omega_{2}^{T} & W_{3}
\end{array}\right) \leq 0,
$$

where

$$
\begin{aligned}
W_{2}= & W_{1}+\gamma_{4}\left(I_{N} \otimes B_{1}^{T}\right)\left(I_{N} \otimes B_{1}\right), \\
\Omega_{2}= & \Omega_{1}+\gamma_{4}\left(I_{N} \otimes B_{1}^{T}\right)\left(I_{N} \otimes B_{2}\right), \\
W_{3}= & \gamma_{4}\left(I_{N} \otimes B_{2}^{T}\right)\left(I_{N} \otimes B_{2}\right) \\
& -I_{N} \otimes \frac{B_{2}+B_{2}^{T}}{2},
\end{aligned}
$$

$W_{1}, \Omega_{1}$ have the same meanings as in Theorem 5.

Proof. Select the same $V_{2}(t)$ as (58) for network (53). By (37) and (64), we get

$$
\begin{aligned}
\mathscr{L} V_{2}( & t)-u^{T}(t) y(t)+\gamma_{4} y^{T}(t) y(t) \\
\leq & \zeta^{T}(t)\left(\begin{array}{cc}
W_{2} & \Omega_{2} \\
\Omega_{2}^{T} & W_{3}
\end{array}\right) \zeta(t) \\
& -2 \beta\left[\left(\frac{a}{1-d} \int_{t-\tau(t)}^{t} e^{T}(h) M e(h) \mathrm{d} h\right)^{((\alpha+1) / 2)}\right. \\
& \left.+\left(\sum_{i=1}^{N} e_{i}^{T}(t) P e_{i}(t)\right)^{((\alpha+1) / 2)}\right] \\
\leq & -2 \beta\left(\frac{a}{1-d} \int_{t-\tau(t)}^{t} e^{T}(h) M e(h) \mathrm{d} h\right. \\
& \left.+\sum_{i=1}^{N} e_{i}^{T}(t) P e_{i}(t)\right)^{((\alpha+1) / 2)} \\
= & -2 \beta V_{2}^{((\alpha+1) / 2)}(t) .
\end{aligned}
$$

Taking the mathematical expectation on (59), we can obtain

$$
\begin{aligned}
& E\left\{u^{T}(t) y(t)\right\}-\gamma_{4} E\left\{y^{T}(t) y(t)\right\} \\
\geq & \frac{E\left\{\mathrm{~d} V_{2}(t)\right\}}{\mathrm{d} t}+2 \beta E\left\{V_{2}^{((\alpha+1) / 2)}(t)\right\} .
\end{aligned}
$$

Therefore, network (53) is finite-time output strictly passive in the sense of expectation under controller (55).

\subsection{Finite-Time Synchronization}

Theorem 8. Assume that a continuous, positive-definite function $\widehat{V}(t)$ satisfies the following inequality:

$$
\varphi_{2}\left(E\|e(t)\|_{2}\right) \leq \widehat{V}(t)
$$

where $\varphi_{2}:[0,+\infty) \longrightarrow[0,+\infty)$ is continuous and strictly monotonically increasing function and $\varphi_{2}(s)$ is positive for $s>0$ with $\varphi_{2}(0)=0$. If network (51) is finite-time passive (finite-time input strictly passive, finite-time output strictly passive) in the sense of expectation with respect to $\widehat{V}(t)$, then SCCN (53) is finite-time synchronized in the sense of expectation under controller (55).

Here we omit the proof of the theorem. The readers can refer to the proof of Theorem 4 .

\section{Numerical Examples}

Example 1. The following SCCNs are discussed:

$$
\begin{aligned}
\mathrm{d} z_{i}(t)= & {\left[f\left(z_{i}(t)\right)+0.7 \sum_{j=1}^{6} G_{i j} \Gamma z_{j}(t)+u_{i}(t)\right.} \\
& \left.+v_{i}(t)\right] \mathrm{d} t+h\left(z_{i}(t)\right) \mathrm{d} \omega(t), \quad i=1,2, \ldots, 6,
\end{aligned}
$$

where $\quad f_{s}(\varsigma)=(1 / 4)(|\varsigma+1|-|\varsigma-1|), s=1,2,3, \Gamma=\operatorname{diag}$ $(0.15,0.05,0.25), h\left(z_{i}(t)\right)=\operatorname{diag}(0.2,0.4,0.2)$,

$$
G=\left(\begin{array}{cccccc}
-0.4 & 0.1 & 0 & 0.1 & 0.2 & 0 \\
0.1 & -0.4 & 0 & 0.2 & 0.1 & 0 \\
0 & 0 & -0.5 & 0.2 & 0.1 & 0.2 \\
0.1 & 0.2 & 0.2 & -0.6 & 0 & 0.1 \\
0.2 & 0.1 & 0.1 & 0 & -0.5 & 0.1 \\
0 & 0 & 0.2 & 0.1 & 0.1 & -0.4
\end{array}\right) .
$$

Obviously, $H(1)$ holds under the condition that $P=I_{3}, \lambda=0.8$, and $\Delta=\operatorname{diag}(0.15,0.14,0.12)$. Choose $A_{2}=$ $\operatorname{diag}(0.6,0.8,0.9)$ and

$$
A_{1}=\left(\begin{array}{lll}
0.3 & 0.2 & 0.1 \\
0.4 & 0.3 & 0.3 \\
0.6 & 0.1 & 0.4
\end{array}\right) \text {. }
$$

Take $Q=\operatorname{diag}\left(0.2 I_{3}, 0.6 I_{3}, 0.7 I_{3}, 0.5 I_{3}, 0.3 I_{3}, 0.4 I_{3}\right)$. According to Theorem 1, the SCCNs (74) can realize finite-time passivity in the sense of expectation under controller (17). Then, we can easily find the parameters $\gamma_{1}=0.0163$ and $\gamma_{2}=0.0300$ satisfying the condition of Theorems 2 and 3 . The simulation results are shown in Figures 1 and 2.

By Theorem 4, network (74) under finite-time output strictly passive can achieve finite-time synchronization. Figure 3 shows the simulation results.

Example 2. The following SCCNs with time-varying delay are discussed: 


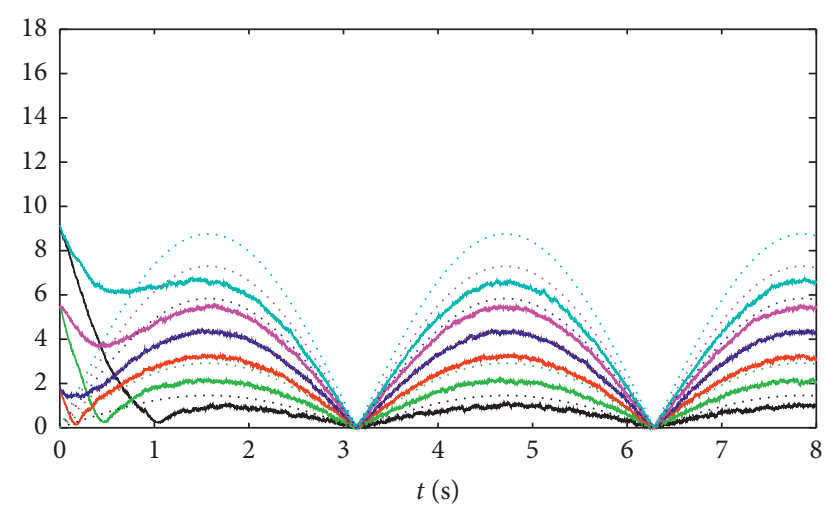

$$
\begin{aligned}
& \ldots \ldots\left\|u_{1}(t)\right\|_{2} \quad \ldots \ldots\left\|u_{4}(t)\right\|_{2} \quad-\left\|y_{1}(t)\right\|_{2} \quad-\left\|y_{4}(t)\right\|_{2} \\
& \left\|u_{2}(t)\right\|_{2} \quad \cdots \cdots\left\|u_{5}(t)\right\|_{2}-\left\|y_{2}(t)\right\|_{2}-\left\|y_{5}(t)\right\|_{2} \\
& \left\|u_{3}(t)\right\|_{2} \quad \ldots \ldots \cdot\left\|u_{6}(t)\right\|_{2}-\left\|y_{3}(t)\right\|_{2}-\left\|y_{6}(t)\right\|_{2}
\end{aligned}
$$

Figure 1: The norms of the input and output vectors $\left\|u_{i}(t)\right\|_{2},\left\|y_{i}(t)\right\|_{2}, i=1,2, \ldots, 6$.

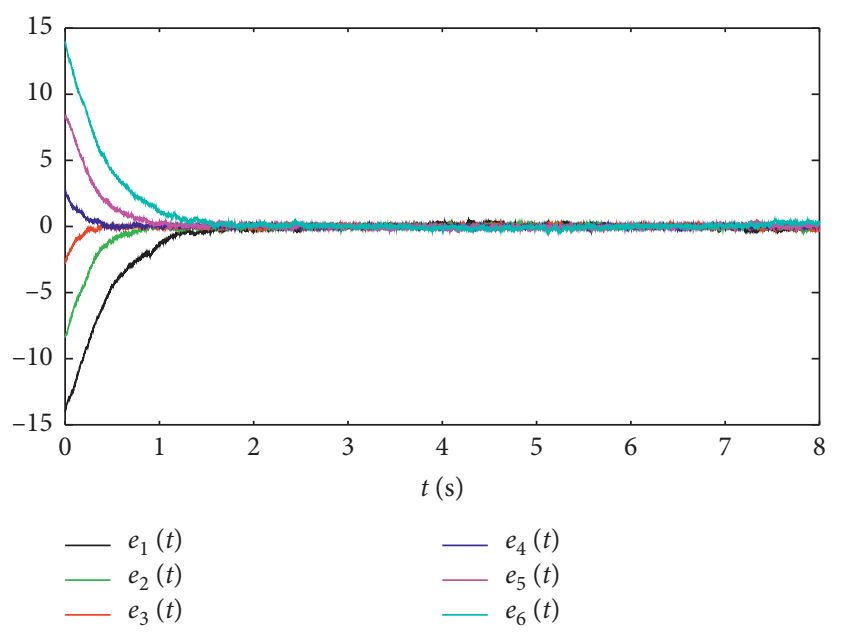

FIgURE 2: The error vectors $e_{i}(t), i=1,2, \ldots, 6$.

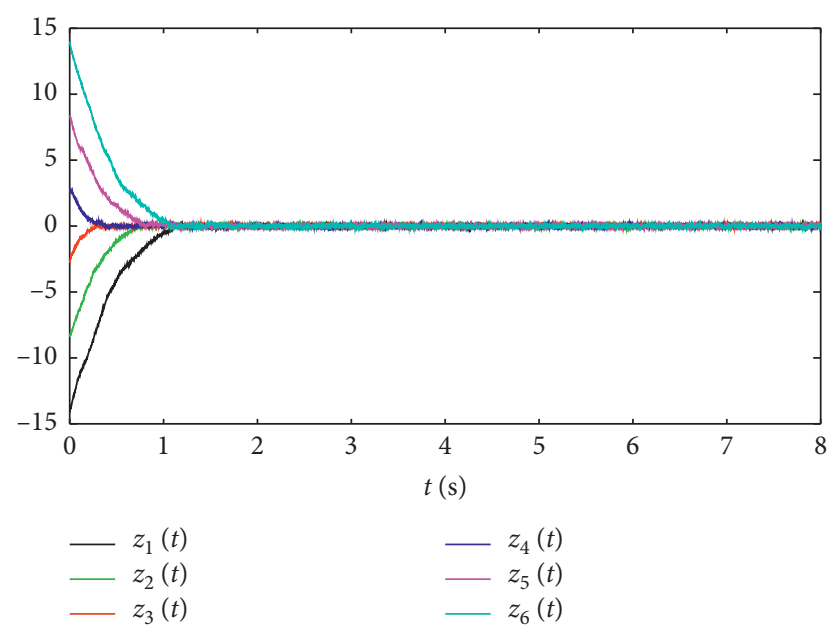

FIgURE 3: The state vectors $z_{i}(t), i=1,2, \ldots, 6$.

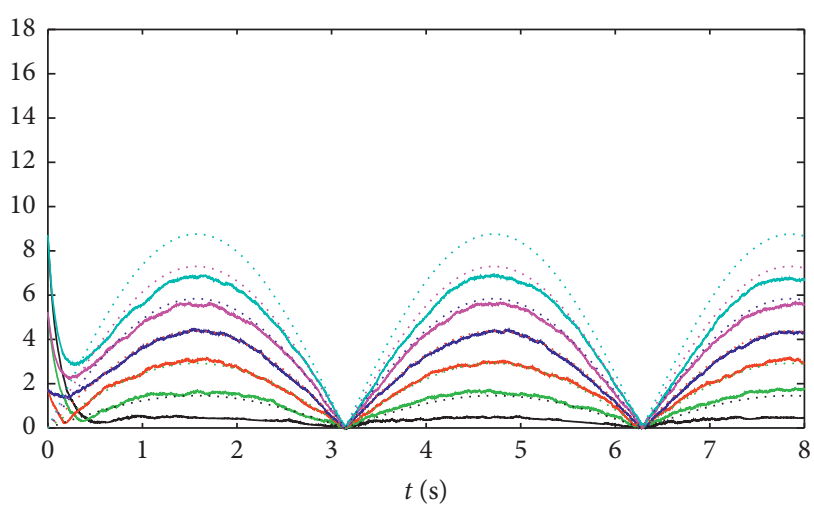

$$
\begin{array}{llll}
\left\|u_{1}(t)\right\|_{2} & \ldots \ldots .\left\|u_{4}(t)\right\|_{2} & -\left\|y_{1}(t)\right\|_{2} & -\left\|y_{4}(t)\right\|_{2} \\
\left\|u_{2}(t)\right\|_{2} & \ldots \ldots .\left\|u_{5}(t)\right\|_{2} & -\left\|y_{2}(t)\right\|_{2} & -\left\|y_{5}(t)\right\|_{2} \\
\left\|u_{3}(t)\right\|_{2} & \ldots \ldots .\left\|u_{6}(t)\right\|_{2} & -\left\|y_{3}(t)\right\|_{2} & -\left\|y_{6}(t)\right\|_{2}
\end{array}
$$

FIgURE 4: The norms of the input and output vectors $\left\|u_{i}(t)\right\|_{2},\left\|y_{i}(t)\right\|_{2}, i=1,2, \ldots, 6$.

$$
\begin{aligned}
\mathrm{d} z_{i}(t)= & {\left[f\left(z_{i}(t)\right)+0.8 \sum_{j=1}^{6} G_{i j} \Gamma z_{j}(t-\tau(t))\right.} \\
& \left.+u_{i}(t)+v_{i}(t)\right] \mathrm{d} t+h\left(z_{i}(t)\right) \mathrm{d} \omega(t), \quad i=1,2, \ldots, 6
\end{aligned}
$$

where $f_{s}(\varsigma)=(1 / 4)(|\varsigma+1|-|\varsigma-1|), s=1,2,3, \quad \Gamma=\operatorname{diag}$ $(0.15,0.15,0.15), \quad h\left(z_{i}(t)\right)=\operatorname{diag}(0.1,0.2,0.2)$. Taking $\tau(t)=0.5-0.5 e^{-t}$, we can get $d=0.5$. The matrix $G$ is chosen as

$$
G=\left(\begin{array}{cccccc}
-0.8 & 0.1 & 0.2 & 0.1 & 0.2 & 0.2 \\
0.1 & -0.5 & 0 & 0.2 & 0.1 & 0.1 \\
0.2 & 0 & -0.7 & 0.2 & 0.1 & 0.2 \\
0.1 & 0.2 & 0.2 & -0.6 & 0 & 0.1 \\
0.2 & 0.1 & 0.1 & 0 & -0.5 & 0.1 \\
0.2 & 0.1 & 0.2 & 0.1 & 0.1 & -0.7
\end{array}\right) .
$$

Obviously, $H(1)$ holds under the condition that $P=I_{3}, \lambda=0.7$, and $\Delta=\operatorname{diag}(0.18,0.16,0.15)$. Choose $B_{2}=$ $\operatorname{diag}(0.6,0.8,0.9)$ and

$$
B_{1}=\left(\begin{array}{lll}
0.6 & 0.4 & 0.3 \\
0.2 & 0.1 & 0.1 \\
0.3 & 0.3 & 0.4
\end{array}\right) \text {. }
$$

Take $Q=\operatorname{diag}\left(0.5 I_{3}, 0.6 I_{3}, 0.7 I_{3}, 0.5 I_{3}, 0.7 I_{3}, 0.8 I_{3}\right)$, $M=I_{6} \otimes \operatorname{diag}(0.1,0.2,0.4)$. According to Theorem 5 , the SCCNs (77) can realize finite-time passivity under controller (55). Then, we can easily find the parameters $\gamma_{3}=0.0132$ and $\gamma_{4}=0.0369$ satisfying the condition of Theorems 6 and 7 . The simulation results are shown in Figures 4 and 5.

By Theorem 8, network (77) under finite-time output strictly passive can achieve finite-time synchronization. Figure 6 shows the simulation results. 


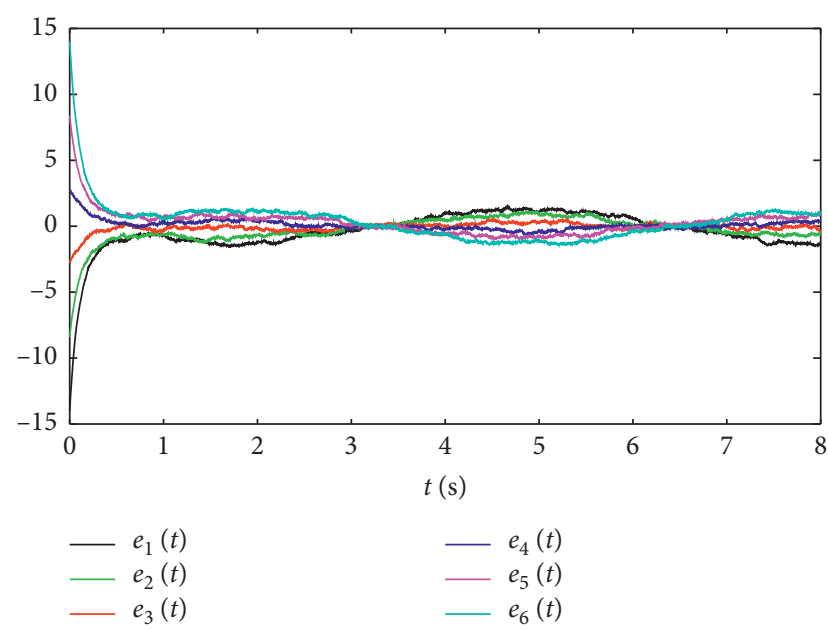

Figure 5: The error vectors $e_{i}(t), i=1,2, \ldots, 6$.

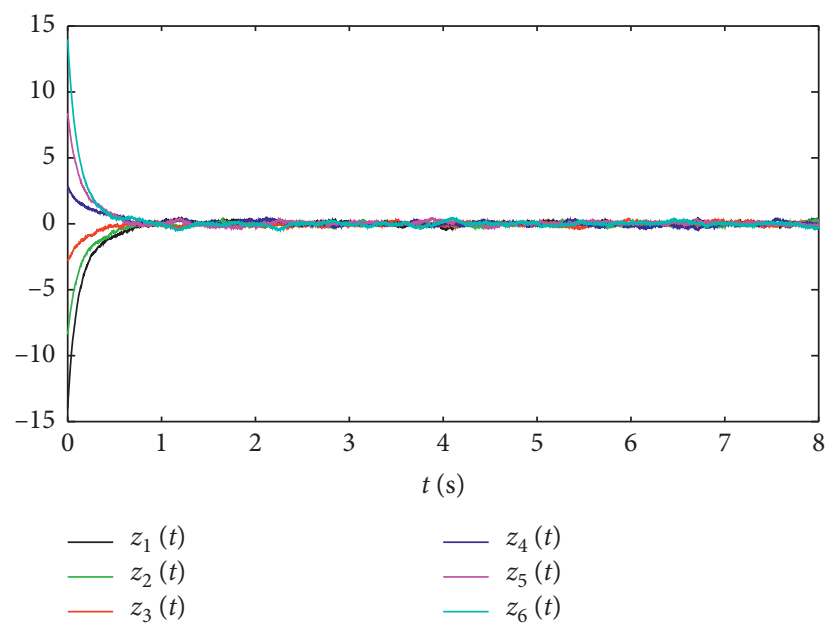

Figure 6: The state vectors $z_{i}(t), i=1,2, \ldots, 6$.

\section{Data Availability}

The data used to support the findings of this study are included within the article.

\section{Conflicts of Interest}

The authors declare that they do not have any commercial or associative interest that represents a conflict of interest in connection with the work submitted.

\section{Acknowledgments}

This study was supported by the National Natural Science Foundation of China under grant nos. 61773285 and 11871371.

\section{References}

[1] J. Ruths and D. Ruths, "Control profiles of complex networks," Science, vol. 343, no. 6177, pp. 1373-1376, 2014.
[2] A. R. Benson, D. F. Gleich, and J. Leskovec, "Higher-order organization of complex net- works," Science, vol. 353, no. 6295, pp. 163-165, 2016.

[3] J. Gao, B. Barzel, and A. Barabsi, "Universal resilience patterns in complex networks," Nature, vol. 530, no. 7590, pp. 307-312, 2016.

[4] J. Liang, Z. Wang, and X. Liu, "Exponential synchronization of stochastic delayed discrete time complex networks," Nonlinear Dynamics, vol. 53, pp. 153-165, 2008.

[5] C. Li, L. Chen, and K. Aihara, "Stochastic synchronization of genetic oscillator networks," BMC Systems Biology, vol. 1, pp. 1-6, 2007.

[6] Y. Wang, Z. Wang, and J. Liang, "A delay fractioning approach to global synchronization of delayed complex networks with stochastic disturbances," Physics Letters A, vol. 372, no. 9, pp. 6066-6073, 2008.

[7] Z. Wang, Y. Wang, and Y. Liu, "Global synchronization for discrete time stochastic complex networks with randomly occurred nonlinearities and mixed time delays," IEEE Transactions on Neural Networks, vol. 21, no. 1, pp. 11-26, 2010.

[8] H. Li and D. Yue, "Synchronization of Markovian jumping stochastic complex networks with distributed time delays and probabilistic interval discrete time varying delays," Journal of Physics A: Mathematical and Theoretical, vol. 43, no. 10, 2010.

[9] X. Lou and B. Cui, "Passivity analysis of integro-differential neural networks with time-varying delays," Neurocomputing, vol. 70, pp. 1071-1078, 2007.

[10] J. Yao, H. O. Wang, Z.-H. Guan, and W. Xu, "Passive stability and synchronization of complex spatio-temporal switching networks with time delays," Automatica, vol. 45, pp. 1721-1728, 2009.

[11] J. L. Wang, H. N. Wu, and L. Guo, "Passivity and stability analysis of reaction-diffusion neural networks with dirichlet boundary conditions," IEEE Transactions on Neural Networks, vol. 22, pp. 2105-2116, 2011.

[12] X. W. Yin, X. N. Song, and M. Wang, "Passive fuzzy control design for a class of nonlinear distributed parameter systems with time-varying Delay," International Journal of Control, Automation and Systems, vol. 18, no. 4, pp. 911-921, 2020.

[13] Z. G. Wu, P. Shi, and J. Chu, "Passivity analysis for discretetime stochastic Markovian jump neural networks with mixed time delays," IEEE Transactions on Neural Networks, vol. 22, no. 10, pp. 1566-1575, 2011.

[14] Q. Song, J. Liang, and Z. Wang, "Passivity analysis of discretetime stochastic neural networks with time-varying delays," Neurocomputing, vol. 72, pp. 1782-1788, 2009.

[15] H. Y. Du, W. S. Sun, G. Hu, and L. H. Qi, "Function projective synchronization of two fractional-order complex dynamical networks," Acta Automatica Sinica, vol. 42, no. 2, pp. 226-234, 2016.

[16] V. Andrieu, B. Jayawardhana, and S. Tarbouriech, "Some results on exponential synchronization of nonlinear systems," IEEE Transactions on Automatic Control, vol. 63, no. 4, pp. 1213-1219, 2018.

[17] D. Zhang, Y. Shen, and J. Mei, "Finite-time synchronization of multi-layer nonlinear coupled complex networks via intermittent feedback control," Neurocomputing, vol. 225, pp. 129-138, 2017.

[18] X. Lu, X. Zhang, and Q. Liu, "Finite-time synchronization of nonlinear complex dynamical networks on time scales via pinning impulsive control," Neurocomputing, vol. 275, pp. 2104-2110, 2018. 
[19] M. Tan, X. Li, and Y. Liu, "Finite-time stability and synchronization of the coupled switched neural networks with nodes of different dimensions," Neural Processing Letters, vol. 49, no. 1, pp. 285-303, 2019.

[20] X. Yang and J. Lu, "Finite-time synchronization of coupled networks with Markovian topology and impulsive effects," IEEE Transactions on Automatic Control, vol. 61, no. 8, pp. 2256-2261, 2016.

[21] E. Moulay and M. Dambrine, "Finite-time stability and stabilization of time-delay systems," System \& Control Letter, vol. 57, no. 7, pp. 561-566, 2008.

[22] J. L. Wang, M. Xu, H. N. Wu, and T. W. Huang, "Finite-time passivity of coupled neural networks with multiple weights," IEEE Transcations on Network Science and Engineering, vol. 5, no. 3, pp. 184-197, 2018.

[23] Y. Tang, "Terminal sliding mode control for rigid robots," Automatica, vol. 34, pp. 51-56, 1998.

[24] X. Huang, W. Lin, and B. Yang, "Global finite-time stabilization of a class of uncertain nonlinear systems," Automatica, vol. 41, pp. 881-888, 2005.

[25] J. L. Wang, H. N. Wu, and S. Y. Ren, Passivity of Complex Dynamical Networks, Springer, Berlin, Germany, 2021.

[26] W. L. Lu and T. P. Chen, "New approach to synchronization analysis of linearly coupled ordinary differential systems," Physica D, vol. 213, no. 2, pp. 214-230, 2006. 